GT2004-53553

\title{
THE TRANSIENT LIQUID CRYSTAL TECHNIQUE: INFLUENCE OF SURFACE CURVATURE AND FINITE WALL THICKNESS
}

\author{
G. Wagner, M. Kotulla*, P. Ott \\ Laboratoire de Thermique Appliquée et de Turbomachine (LTT) \\ Swiss Federal Institute of Technology \\ $\mathrm{CH}-1015$ Lausanne, Switzerland \\ B. Weigand, J. von Wolfersdorf \\ Institute of Aerospace Thermodynamics (ITLR) \\ University of Stuttgart \\ D-70569 Stuttgart, Germany
}

\begin{abstract}
The transient liquid crystal technique is nowadays widely used for measuring the heat transfer characteristics in gas turbine applications. Usually, the assumption is made that the wall of the test model can be treated as a flat and semi-infinite solid. This assumption is correct as long as the penetration depth of the heat compared to the thickness of the wall and to the radius of curvature is small. However, those two assumptions are not always respected for measurements near the leading edge of a blade. This paper presents a rigorous treatment of the curvature and finite wall thickness effects. The unsteady heat transfer for a hollow cylinder has been investigated analytically and a data reduction method taking into account curvature and finite wall thickness effects has been developed. Experimental tests made on hollow cylinder models have been evaluated using the new reduction method as well as the traditional semi-infinite flat plate approach and a third method that approximately accounts for curvature effects. It has been found that curvature and finite thickness of the wall have in some cases a significant influence on the obtained heat transfer coefficient. The parameters influencing the accuracy of the semi-infinite flat plate model and the approximate curvature correction are determined and the domains of validity are represented.
\end{abstract}

\footnotetext{
* Present address: Institut für Luftfahrtantriebe (ILA)

University of Stuttgart

D-70569 Stuttgart Germany
}

\section{NOMENCLATURE}

\section{Symbols}

$B i \quad$ Biot number

$b$ radius ratio

c specific heat capacity

$d \quad$ wall thickness

D diameter of cylinder

$h \quad$ heat transfer coefficient

$h_{f p} \quad$ heat transfer coefficient from flat plate analysis Eq. (1)

$h_{R} \quad$ heat transfer coefficient from simplified model Eq. (21)

$k \quad$ thermal conductivity

$L_{x} \quad$ turbulent length scale

Ma Mach number

$\mathrm{Nu} \quad$ Nusselt number

$r \quad$ radius

$\widetilde{r} \quad$ dimensionless radial coordinate

$R \quad$ radius of curvature

Re Reynolds number

$s \quad$ coordinate at surface from stagnation point

$T$ temperature

$t$ time

$\mathrm{Tu} \quad$ turbulence level

\section{Greek}

$\alpha \quad$ thermal diffusivity

$\lambda \quad$ eigenvalues

$\rho$ density

$\Theta \quad$ dimensionless temperature

$\sigma \quad$ curvature parameter (1-cylinder, 2-sphere)

$\tau \quad$ dimensionless time 


\section{Subscripts}

$\begin{array}{ll}c & \text { coolant } \\ d & \text { based on wall thickness } \\ f p & \text { evaluated with semi-infinite flat plate assumption } \\ g & \text { hot gas } \\ i & \text { initial } \\ R & \text { based on radius of curvature } \\ t o t & \text { total } \\ w & \text { wall } \\ 1 & \text { inner wall } \\ 2 & \text { outer wall }\end{array}$

\section{INTRODUCTION}

The demand for gas turbines with improved efficiency and higher power output has led to a continuous increase of the turbine inlet temperature. Consequently, turbine components such as blades and platforms are exposed to ever-higher thermal loads. To guarantee safe operating conditions and acceptable blade life the designers have to optimize the cooling systems and to predict with sufficient precision the blade temperatures. To fulfill those tasks, detailed and accurate knowledge of the heat transfer characteristics of the blades are required.

Transient techniques have been successfully and widely used to measure the heat transfer coefficients on both internal and external surfaces of turbine blades (e.g. Clifford et al. (1983), Guo et al. (1995) and Drost and Bölcs (1998)). Ireland and Jones (1985) used for the first time liquid crystals together with the transient technique. They measured the heat transfer coefficient in blade cooling passages with high spatial resolution, and showed the importance of the method for gas turbine applications. The transient experiment is usually generated by a step change in the gas temperature. The evolution of the surface temperature during the transient test is measured and compared with the predictions of a one-dimensional heat conduction model for a prescribed heat transfer coefficient. This heat conduction model generally considers the wall of the test object as a flat, semi-infinite solid. This assumption is correct as long as the penetration depth of the heat compared to the thickness of the wall and to the radius of curvature is small. Schulz and Jones (1973) evaluated that the semi-infinite model holds if the duration of the experiment is smaller than $t_{\max }=$ $d^{2} / 16 \alpha$. More recently, Vogel and Weigand (2001) compared the semi-infinite model with a more complex finite model and showed that this criterion could be extended to $t_{\max }<d^{2} / 4 \alpha$. Buttsworth and Jones (1997) proposed a simple curvature correction based on a semi-infinite assumption and valid for $t_{\max }$ $<<R^{2} / \alpha$. However, on thin and highly curved walls like in the leading edge region of a film-cooled turbine blade, those limitations on $t_{\max }$ might be violated leading to incorrect heat transfer measurements. In this paper, an exact heat conduction model taking into account the finite thickness of the wall and the curvature is presented. The difference between, the semi-infinite flat plate approach, Buttsworth and Jones's curvature correction and the exact solution are shown for typical experimental situations.

To verify the theoretical considerations, transient liquid crystal experiments were carried out on hollow cylinders with different wall thickness. Heat transfer coefficients are obtained by evaluating the measurement data with the different heat conduction models. The results are compared with recent correlations for convective heat transfer on a cylinder in crossflow (Dullenkopf and Mayle 1994, Van Fossen at al. 1995 and Oo and Ching 2001). The rigorous treatment of wall thickness and curvature effects presented in this paper should help to further improve accurate transient liquid crystal measurements.

\section{EXPERIMENTAL SETUP AND MEASURING TECHNIQUE}

\section{Experimental Setup}

A free jet facility supplied by a continuously running compressor was used for this study (Fig. 1). The diameter of the free jet nozzle was $150 \mathrm{~mm}$. A square-meshed turbulence grid with a mesh size of $15 \mathrm{~mm}$ and a bar width of $3 \mathrm{~mm}$ was installed. The turbulence intensity at the location of the cylinder leading edge was about $T u=7 \%$. The transient experiments were performed at two Mach numbers, $M a=0.11$ and $M a=0.14$ with a gas total temperature of $45^{\circ} \mathrm{C}$. A non-dimensional integral length scale of $\mathrm{L}_{\mathrm{x}} / D=0.30$ at $M a=0.14$ was measured by Reiss (2000) using a hot-wire probe.

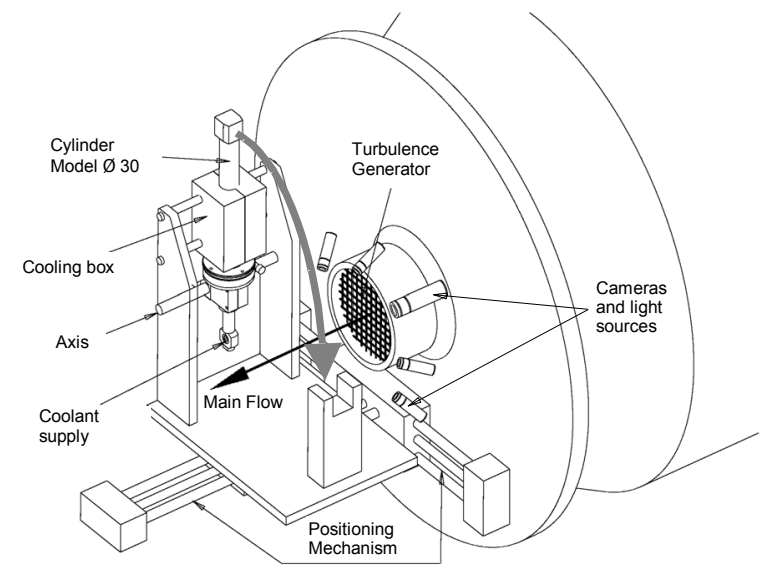

Figure 1: Preconditioning of the cylinder model and rapid insertion mechanism

A rapid insertion mechanism with a pre-cooling box was used to generate the transient experiment. The Perspex hollow cylinder model was pre-cooled and rapidly exposed to the hot cross flow. To investigate the influence of finite wall thickness and internal convection on transient liquid crystal measurements, the cylinder was internally cooled. Coolant temperatures varied between 0 and $10^{\circ} \mathrm{C}$. The cooling air for the preconditioning box and the internal coolant were provided 
by the same source of dried cold air. However, as different temperatures were required, individual mass flow and temperature regulation was possible.

Before the transient experiment, the model was in a vertical position and locked in the two-parts cooling box for preconditioning the test section as shown in Fig. 1. During the preconditioning period the internal cooling was bypassed so that there was no coolant flow inside the cylinder. When the initial temperature was homogenous and at the desired level (typically $-5^{\circ} \mathrm{C}$ ), the cylinder could be inserted into the main flow for a transient test. Once the insertion mechanism was released, the preconditioning box opened and two springs pulled the cylinder, which pivoted rapidly into the free jet. At the same time, the coolant valve was triggered and the internal cooling was activated. A rubber stop locked the cylinder in the horizontal position. The time needed for the insertion (from the opening of the cooling box until the cylinder stops in its final position) was less than $0.15 \mathrm{~s}$.

Three hollow cylinders with the same external diameter of $30 \mathrm{~mm}$ but with different wall thicknesses of $2 \mathrm{~mm}, 5 \mathrm{~mm}$ and $10 \mathrm{~mm}$ were tested. The models were made out of Plexiglas XT because of its low thermal conductivity. To measure the initial temperature, thin thermocouples were glued on both the external and the internal surfaces at $60^{\circ}, 0^{\circ}$ and $-60^{\circ}$ (Fig. 2). Preliminary pre-conditioning tests have been done with a Perspex model containing 8 thermocouples. It was found that the pre-cooling setup allowed temperature uniformity of the test region within $\pm 0.2^{\circ} \mathrm{C}$. The coolant temperature during the transient test was measured with a thermocouple. At the external surface of the cylinder models, a thin and uniform liquid crystal coating was applied. The liquid crystals used for this study react at $36^{\circ} \mathrm{C}$ and show the whole visible spectrum in a $0.7 \mathrm{~K}$ temperature range.

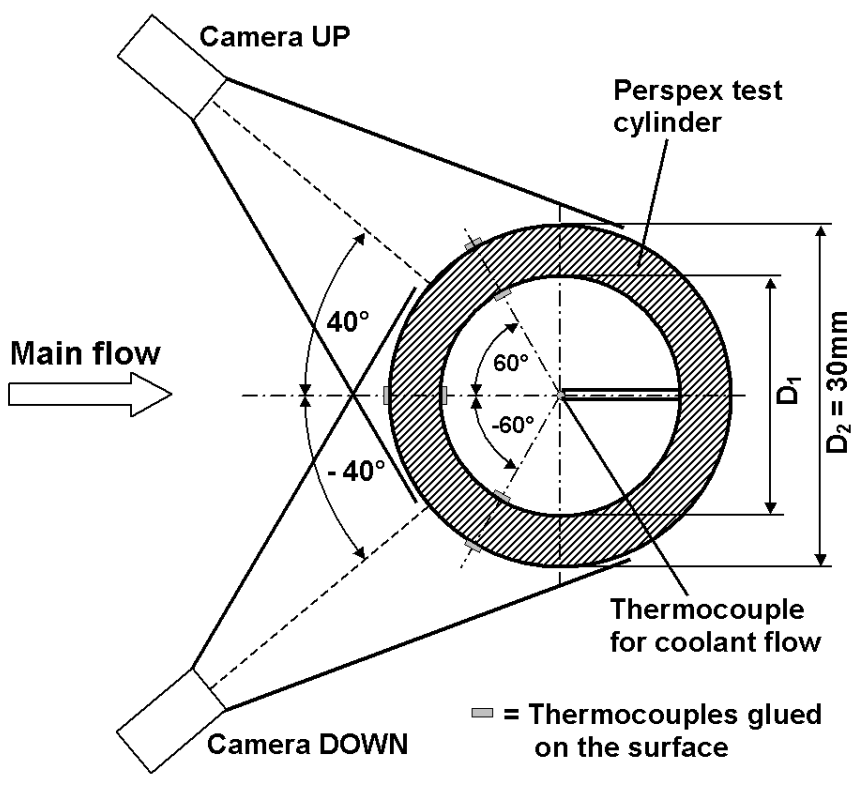

Figure 2: Configuration of hollow cylinder model
Two $25 \mathrm{~Hz}$ color CCD cameras were installed for recording the color play of the liquid crystals on both side of the cylinder. The viewing angles from the stagnation line were $-40^{\circ}$ and $40^{\circ}$ (Fig.2). A check of the symmetry of the results around the leading edge was then possible. Six light sources were fitted around the nozzle of the free jet in order to obtain a homogenous illumination of the test section.

\section{Measuring Technique}

In comparison to steady state measurements, transient techniques are of short duration and allow performing many experiments in a reasonable measurement time. With the transient technique the undesirable lateral conduction inside the model is much smaller than the conduction normal to the surface and does not affect the results. Finally, no heater elements need to be fitted on the test surface and complex geometries can be investigated. Different techniques can be used to generate the step change in the gas temperature. Ireland and Jones (1985) employed fast acting valves to suddenly activate a hot air flow through the test section, while Wang et al. (1996) used electrical heater grids in the main flow. In the frame of this work a pre-cooled test model was rapidly exposed to a hot cross flow with a rotating insertion mechanism (Fig. 1).

Temperature evolution of the model surface is measured by a single layer of narrow-band thermo-chromic liquid crystals. The liquid crystal signal appears first in the area of high heat transfer moves towards the lower heat transfer regions and disappears when the surface temperature is higher than the reacting temperature of the liquid crystals. The video sequences of the transient tests are recorded on a digital support and transferred to a computer for the data reduction. An image processing based on a hue filtering detects the apparition time of the liquid crystal signal for every point on the 2D surface. The resolution is only limited by the data acquisition system. The temperature corresponding to the liquid crystal signal is obtained by a calibration process. This hue capturing technique is further described by Ireland and Jones (2000).

\section{Data Analysis}

The data analysis is based on the theory of onedimensional heat conduction with a boundary condition of the third kind (convective heat exchange) at the outer surface. The transient experiments are usually evaluated using the model of a semi-infinite flat plate with a step change in gas temperature, for which the wall temperature response is given by:

$$
\Theta_{w}=\frac{T_{w}-T_{i}}{T_{g}-T_{i}}=1-\exp \left(\frac{h_{f p}{ }^{2} t}{k \rho c}\right) \operatorname{erfc}\left(\frac{h_{f p} \sqrt{t}}{\sqrt{k \rho c}}\right)
$$

Knowing the time apparition of a specific temperature for a point on the surface, equation (1) can be solved in order to obtain the local heat transfer coefficient $h_{f p}$, where the subscript indicates the use of the flat plate analysis. This model has the advantage of easy and time effective evaluation. 
For the semi-infinite approach the measurement time has to be limited as heat is only allowed to penetrate a small fraction of the wall thickness. The effects of finite wall thickness were evaluated by Vogel and Weigand (2001) using the analytical solution of a finite flat plate with convection boundary conditions on both sides. For the investigation of heat transfer on curved surfaces with finite wall thickness, the analysis needs to be done for a hollow cylinder. Therefore the unsteady, onedimensional heat conduction in the geometry shown in Fig. 3 is investigated analytically. At the inner and the outer surface heat is exchanged by convection.

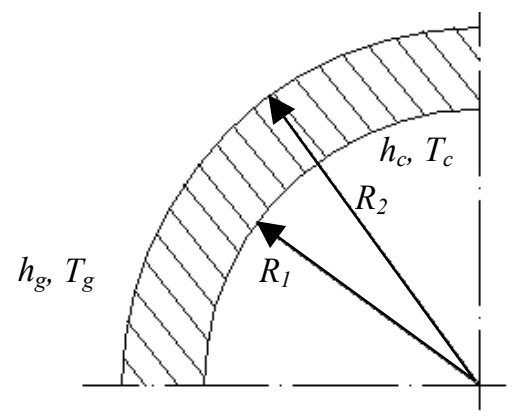

Figure 3: Geometry under consideration

Assuming one-dimensional transient heat conduction in the solid and under the assumption that the properties of the solid are constant, the energy equation simplifies to

$$
\rho c \frac{\partial T}{\partial t}=\frac{k}{r} \frac{\partial}{\partial r}\left(r \frac{\partial T}{\partial r}\right)
$$

with the following boundary conditions:

$$
\begin{aligned}
& t=0: \quad T=T_{i} \\
& r=R_{2}: \quad h_{g}\left[T_{g}-T\left(r=R_{2}\right)\right]-\left.k \frac{\partial T}{\partial r}\right|_{r=R_{2}}=0 \\
& r=R_{1}: \quad h_{c}\left[T_{c}-T\left(r=R_{1}\right)\right]+\left.k \frac{\partial T}{\partial r}\right|_{r=R_{1}}=0
\end{aligned}
$$

Introducing the following dimensionless quantities into Eqs. (2) and (3):

$$
\begin{aligned}
& \tilde{r}=\frac{r}{R_{1}}, \Theta=\frac{T-T_{i}}{T_{g}-T_{i}}, \tau=\frac{t \alpha}{R_{1}^{2}} \text { with } \alpha=\frac{k}{\rho c}, \\
& B i_{g}=\frac{h_{g} R_{2}}{k}, B i_{c}=\frac{h_{c} R_{1}}{k}, \Theta_{c}=\frac{T_{c}-T_{g}}{T_{i}-T_{g}}, b=\frac{R_{2}}{R_{1}}
\end{aligned}
$$

results in

$$
\begin{aligned}
& \frac{\partial \Theta}{\partial \tau}=\frac{1}{\tilde{r}} \frac{\partial}{\partial \tilde{r}}\left(\tilde{r} \frac{\partial \Theta}{\partial \tilde{r}}\right) \\
& \tau=0: \quad \Theta=0 \\
& \widetilde{r}=b: \quad \frac{B i_{g}}{b}[1-\Theta(\widetilde{r}=b)]-\left.\frac{\partial \Theta}{\partial \widetilde{r}}\right|_{\tilde{r}=b}=0 \\
& \widetilde{r}=1: \quad B i_{c}\left[1-\Theta_{c}-\Theta(\widetilde{r}=1)\right]+\left.\frac{\partial \Theta}{\partial \widetilde{r}}\right|_{\tilde{r}=1}=0
\end{aligned}
$$

The above given problem has a non-homogeneous boundary condition for $\tilde{r}=1$. Therefore we split the solution into two individual parts:

$$
\Theta=\Theta_{S}+\Theta_{T}
$$

where $\Theta_{S}$ denotes the steady-state temperature distribution of the problem and $\Theta_{T}$ describes the transient part. The steadystate part is given by

$$
\Theta_{S}=A \ln \tilde{r}+B
$$

with

$$
A=\frac{\Theta_{c}}{\ln b+\frac{1}{B i_{g}}+\frac{1}{B i_{c}}}, \quad B=1-\frac{\Theta_{c}\left[\ln b+\frac{1}{B i_{g}}\right]}{\ln b+\frac{1}{B i_{g}}+\frac{1}{B i_{c}}}
$$

For the transient problem, one has to solve the following equations

$$
\begin{aligned}
& \frac{\partial \Theta_{T}}{\partial \tau}=\frac{1}{\widetilde{r}} \frac{\partial}{\partial \widetilde{r}}\left(\widetilde{r} \frac{\partial \Theta_{T}}{\partial \widetilde{r}}\right) \\
& \tau=0: \quad \Theta_{T}=1-A \ln \widetilde{r}-B \\
& \widetilde{r}=b: \quad B i_{g} \Theta_{T}(\widetilde{r}=b)+\left.\frac{\partial \Theta_{T}}{\partial \widetilde{r}}\right|_{\widetilde{r}=b}=0 \\
& \widetilde{r}=1: \quad-B i_{c} \Theta_{T}(\widetilde{r}=1)+\left.\frac{\partial \Theta_{T}}{\partial \widetilde{r}}\right|_{\widetilde{r}=1}=0
\end{aligned}
$$

A solution of these equations can be obtained by using the method of separation of variables. Inserting the expression

$$
\Theta_{T}=T(\tau) R(\widetilde{r})
$$

into Eqs. (10) and (11) results in

$$
T_{n}=C_{n 1} \exp \left(-\lambda_{n}{ }^{2} \tau\right)
$$


and for the eigenfunctions $R_{n}(\widetilde{r})$ the following eigenvalue problem is obtained

$$
\begin{aligned}
& R_{n}{ }^{\prime \prime}+\frac{1}{\tilde{r}} R_{n}{ }^{\prime}+\lambda_{n}{ }^{2} R_{n} \\
& \widetilde{r}=b: \quad B i_{g} R_{n}(b)+R_{n}{ }^{\prime}(b)=0 \\
& \widetilde{r}=1: \quad-B i_{c} R_{n}(1)+R_{n}{ }^{\prime}(1)=0
\end{aligned}
$$

Eqs. (14) and (15) have the solution

$$
R_{n}(\tilde{r})=E_{n}\left\{J_{0}\left(\lambda_{n} \tilde{r}\right)-\frac{\lambda_{n} J_{1}\left(\lambda_{n}\right)+B i_{c} J_{0}\left(\lambda_{n}\right)}{\lambda_{n} Y_{1}\left(\lambda_{n}\right)+B i_{c} Y_{0}\left(\lambda_{n}\right)} Y_{0}\left(\lambda_{n} \tilde{r}\right)\right\}
$$

where $J_{0}, J_{l}, Y_{0}$ and $Y_{l}$ are Bessel's functions. The eigenvalues are given by the transcendental equation

$$
\begin{aligned}
& {\left[B i_{g} J_{0}\left(\lambda_{n} b\right)-\lambda_{n} J_{1}\left(\lambda_{n} b\right)\right]\left[B i_{c} Y_{0}\left(\lambda_{n}\right)+\lambda_{n} Y_{1}\left(\lambda_{n}\right)\right]} \\
& -\left[B i_{g} Y_{0}\left(\lambda_{n} b\right)-\lambda_{n} Y_{1}\left(\lambda_{n} b\right)\right]\left[B i_{c} J_{0}\left(\lambda_{n}\right)+\lambda_{n} J_{1}\left(\lambda_{n}\right)\right]=0
\end{aligned}
$$

The solution for the transient part of the problem is given by

$$
\Theta_{T}=\sum_{n=1}^{\infty} D_{n} R_{n}(\widetilde{r}) \exp \left(-\lambda_{n}{ }^{2} \tau\right)
$$

with

$$
D_{n}=-\frac{\int_{1}^{b}[B+A \ln \widetilde{r}] \widetilde{r} R_{n}(\widetilde{r}) d \widetilde{r}}{\int_{1}^{b} \widetilde{r} R_{n}^{2}(\widetilde{r}) d \widetilde{r}}
$$

The surface temperature $\Theta(\widetilde{r}=b, \tau)=\Theta_{S}+\Theta_{T}$ is finally

$$
\Theta_{w}(\tau)=\frac{T_{w}-T_{i}}{T_{g}-T_{i}}=A \ln (b)+B+\sum_{n=1}^{\infty} D_{n} R_{n}(b) \exp \left(-\lambda_{n}{ }^{2} \tau\right)
$$

The above obtained solution can also be derived by using the Laplace transform technique and the reader is referred to Carslaw and Jäger (1992) for this type of approach. Eq. (20) will be used for the hollow cylinder.

\section{Uncertainty analysis}

The uncertainties in the measurement of the temperatures $T_{w}, T_{i}, T_{g}$ and in the detection of the liquid crystal apparition time $t$ result in an error on the obtained heat transfer coefficient. This error appears to be bigger for high heat transfer regions like the leading edge, which can be explained by a shorter apparition time and then a higher relative error $\Delta t / t$. Table 1 shows the measurement errors of the experiments carried out in the frame of this study.
For the semi-infinite case, an error analysis as detailed by Ireland (1987) has been performed on Eq. (1). The first 4 errors from Table 1 together with reasonable uncertainties on the material properties indicated an error on the obtained heat transfer coefficient of $5.7 \%$ for the leading edge region.

\begin{tabular}{|l|l|l|}
\hline Quantity & Typical value & Measurement error \\
\hline$T_{g}$ & $318.5[\mathrm{~K}]$ & $\pm 0.15[\mathrm{~K}]$ \\
\hline$T_{i}$ & $268[\mathrm{~K}]$ & $\pm 0.75[\mathrm{~K}]$ \\
\hline$T_{w}$ & $309[\mathrm{~K}]$ & $\pm 0.2[\mathrm{~K}]$ \\
\hline$t$ & $15-60[\mathrm{~s}]$ & $\pm 0.1[\mathrm{~s}]$ \\
\hline$T_{c}$ & $280[\mathrm{~K}]$ & $\pm 7[\mathrm{~K}]$ \\
\hline$h_{c}$ & $13-80\left[\mathrm{~W} / \mathrm{m}^{2} \mathrm{~K}\right]$ & $\pm 50 \%$ \\
\hline
\end{tabular}

Table 1: Measurements errors

An analytical error analysis being too complex for the exact solution (Eq. (20)), a numerical parameter study has been performed. As internal convection is here taken into account, the uncertainties of the internal coolant temperature $T_{c}$ and heat transfer coefficient $h_{c}$ have to be considered. It appeared that $T_{c}$ and $h_{c}$ have only little influence in the obtained heat transfer coefficient. Even with large uncertainties of $7 \mathrm{~K}$ for $T_{c}$ and $50 \%$ for $h_{c}$, the error on the heat transfer coefficient were within $5.5 \%$ for $h_{g}=345 \mathrm{~W} / \mathrm{m}^{2} \mathrm{~K}$ (leading edge region) and $4.5 \%$ for $h_{g}=170 \mathrm{~W} / \mathrm{m}^{2} \mathrm{~K}$. Those uncertainties are due to the errors in the measurement of the different experimental parameters but do not include the error due to the data analysis, which are treated in the next chapter.

\section{EFFECTS OF WALL CURVATURE, THICKNESS AND BACKSIDE HEAT TRANSFER}

\section{Simplified model- Effect of wall curvature}

For curved walls Buttsworth and Jones (1997) used Laplace Transforms and provided in similarity to Eq. (1) a simplified approximate solution valid for $\tau_{R}=\alpha t / R^{2}<<1$, which is given as:

$$
\Theta_{w}=\frac{1}{1 \pm \frac{\sigma k}{2 h_{R} R}}\left[1-\exp \left(\frac{\left(h_{R} \pm \frac{\sigma k}{2 R}\right)^{2} t}{k \rho c}\right) \operatorname{erfc}\left(\frac{\left(h_{R} \pm \frac{\sigma k}{2 R}\right) \sqrt{t}}{\sqrt{k \rho c}}\right)\right]
$$

where $\sigma=1$ for a cylinder, $\sigma=2$ for a sphere, the + sign for concave surfaces and the -sign for convex surfaces.

Using the approximate solution (Eq. (21)) for the data evaluation of the liquid crystal indications, the determined heat transfer coefficient $h_{R}$ takes approximately into account the effect of wall curvature. It is the aim of this section to show the 
influence of curvature and the range of applicability of Eqs. (1) and (21). In the case of large radius of curvature $(R \rightarrow \infty)$, Eq. (21) reduces to Eq. (1) and the determined heat transfer coefficient is $h_{g}=h_{f p}=h_{R}$.

Analysing a transient liquid crystal experiment involves determining the heat transfer coefficient corresponding to the apparition of the dimensionless temperature $\Theta_{w}$ at time $t$. The value of the heat transfer coefficient determined from a transient experiment on a convex surface will be higher using the flat plate model (Eq. (1)) than using Eq. (21), which is $h_{R}<h_{f p}$. For a concave surface one obtains $h_{R}>h_{f p}$. This is shown in Fig. 4 for cylindrical convex and concave surfaces. For this comparison the following procedure was applied. First the heat transfer coefficient on a curved surface $h_{R}$ is prescribed and Eq. (21) is used to evaluate the wall temperature history. Then for chosen liquid crystal indication temperatures and therefore different $\Theta_{w}$ Eq. (1) is solved to obtain $h_{f p}$.

The ratio $h_{R} / h_{f p}$ is given in Fig. 4 for different Biot numbers based on $h_{f p}$ and R. Figure 4 shows that for Biot numbers usually found in transient experiments (typically $B i_{f p, R}>10$ ) the curvature effect can lead to errors up to $10 \%$. For shorter measurement times (lower $\Theta_{\mathrm{w}}$ ) the differences are smaller which needs to be considered in the experimental layout. Analysing some published transient experiments on cylindrical surfaces (e.g. Ekkad and Han (1998), Butler and Baughn (1994), Baughn et al. (1989)) the influence of curvature for the used experimental parameters was found to be less than $3 \%$. The curvature influence needs to be considered especially for small curvature radius tests as found e.g. in the leading edge area of gas turbine blades using cascade heat transfer experiments.

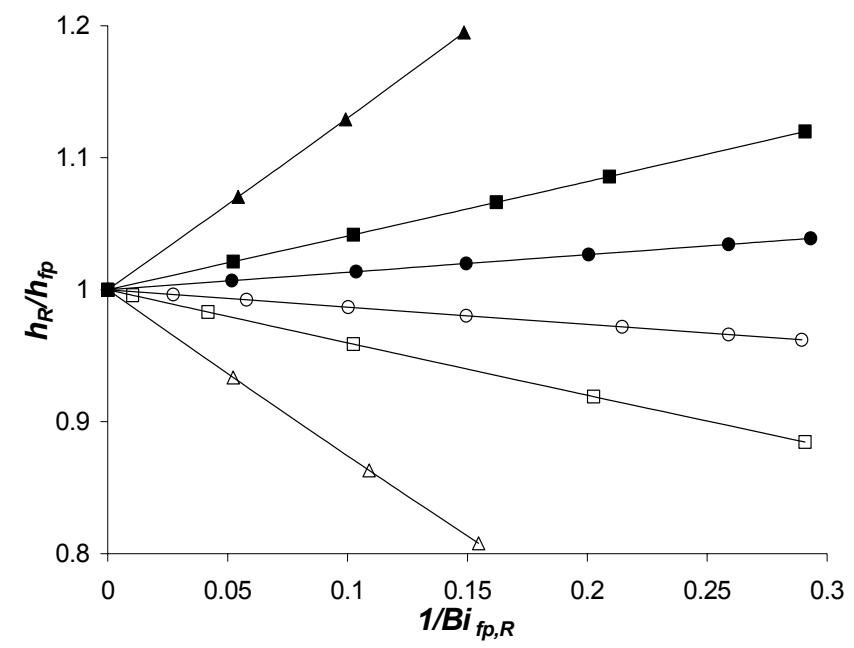

Figure 4: Effect of surface curvature using comparison between Eqs. (1) and (21)

Convex: $\square \Theta_{w}=0.75, \square-\Theta_{w}=0.50, \square-\Theta_{w}=0.25$

Concave: $\multimap \Theta_{w}=0.75, \rightarrow-\Theta_{w}=0.50, \rightarrow-\Theta_{w}=0.25$

Using the exact solution (Eq. (20)) to calculate the temperature history data for a prescribed heat transfer coefficient $h_{g}$ and analysing the data for different time with the approximate solution (Eq. (21)) in order to obtain $h_{R}$ reveals the error of the approximate solution. As given by Buttswort and Jones (1997), the ratio $h_{R} / h_{g}$ can be represented versus $\tau_{R}$ for different Biot numbers based on $h_{g}$ and $R$. This is shown in Fig. 5 for the convex and concave cylinders.

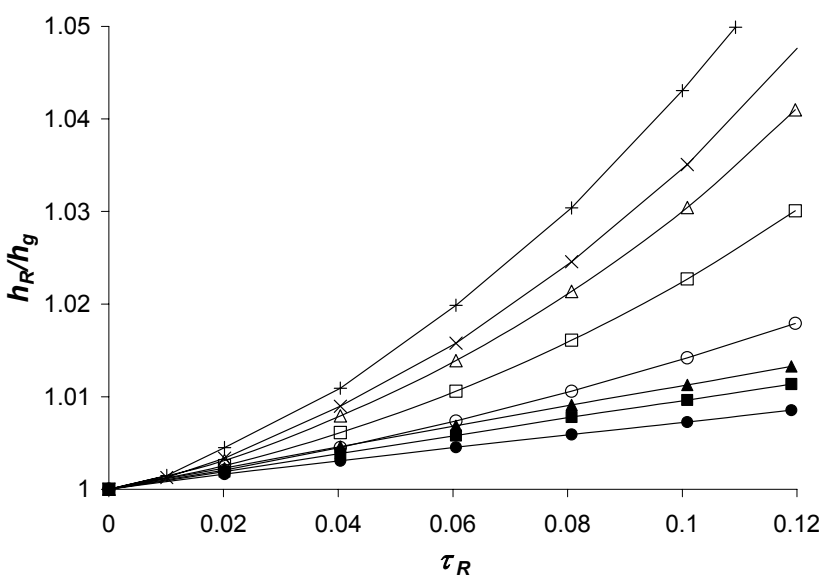

Figure 5: Deviation of the approximate solution Eq. (21) from the exact solution Eq. (20).

Convex: $\square B i_{g, R}=1, \square-\square i_{g, R}=5, \square B i_{g}, R=10, \longleftarrow B i_{g, R}=15$

$+B i_{g, R}=30$

Concave: $\left.\because B i_{g, R}=1, \rightarrow B i_{g, R}=5, \multimap B i_{g, R}=10\right)$

For the cases investigated it is shown that the approximate solution (Eq. (21)) describes the wall temperature history with sufficient accuracy for

$$
\tau_{R}=\frac{\alpha t}{R^{2}}<0.02
$$

If Eq. (22) is respected, the difference of the evaluated heat transfer coefficient with Eq. (21) to the prescribed value is less than $1 \%$. This is important in data evaluation procedures of transient experiments, since Eq. (21) is much easier to evaluate than the exact solution and existing data reduction software can easily be adopted to curved walls due to the similarity of Eqs. (1) and (21). Further if experiments are made with a time varying gas temperature, Eq. (21) can easily be adapted to this using the superposition method as usually applied for flat surfaces (e.g. Ekkad and Han (2000)).

The importance to take the curvature into account and the range of validity for the flat plate and the approximate solution is illustrated in Fig. 6. This figure shows combinations of $\tau_{R}$ and $B i_{g, R}$ for which the differences in evaluated heat transfer coefficients are below specific errors. Below the uppermost curve (no symbols) the heat transfer coefficient evaluated from the approximate solution is within $1 \%$ to the exact value. Using the same accuracy for the flat plate solution the lowest curve is obtained. Increasing the allowable error in the evaluated heat transfer coefficient the region for the flat plate solution is increased. 


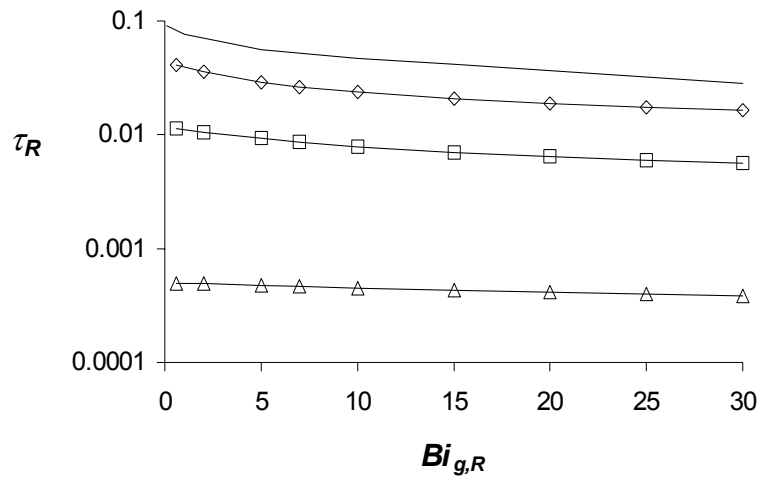

Figure 6: Regions of validity for approximate (Eq. (21)) and flat plate (Eq. (1)) solutions depending on allowable error:

exact - approximate: $-1 \%$

approximate - flat plate: $\longleftarrow 1 \%, \square-5 \%, \multimap 10 \%$

\section{Effect of wall thickness}

For film cooling experiments with showerhead cooling the test models have to have a finite thickness to simulate the correct hole length-to-diameter ratio. This can lead to thin wall models, where the assumption of a semi-infinite solid might not be correct. Schultz and Jones (1973) give a criterion for the maximum test time related to the thermal penetration distance of $\tau_{d}=\alpha t / d^{2}=1 / 16$. This criterion is very severe. As shown by Vogel and Weigand (2001), for a flat plate with typical Biotnumbers the error in the determined heat transfer coefficient is small and the semi-infinite assumption can be considered valid as long as:

$$
\tau_{d}=\frac{\alpha t}{d^{2}}<\frac{1}{4}
$$

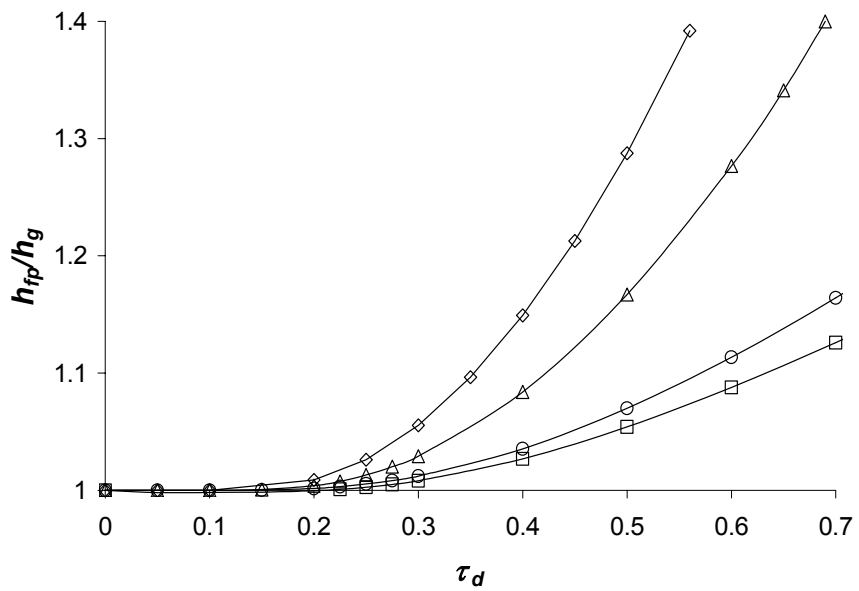

Figure 7: effect of finite wall thickness for flat plate $\square B i_{g, d}=0.5, \multimap B i_{g, d}=1, \longleftarrow B i_{g, d}=5, \leadsto B i_{g, d}=10$

This is shown in Fig. 7. Here a wall of finite thickness with an adiabatic backside is considered. Using the analytical solution (e.g. Vogel and Weigand (2001), Carslaw and Jaeger (1992)) with $B i_{c}=0$ the time temperature history for several gas side Biot-numbers $B i_{g, d}=h_{g} / k$ and wall thicknesses can be calculated. Here the wall thickness is used in the Biot number as appropriate dimension. Evaluating the wall temperature history using Eq. (1) leads to deviations depending on the Biotnumber and the dimensionless time considered.

Since the approximate solution for the curved wall (Eq. (21)) is based on the assumption, that the heat penetrates only a relatively small distance into the wall, it is expected, that this behaviour is similar for a curved wall.

Calculating the time temperature history with the exact solution Eq. (20) and analysing the wall temperature evolution using Eq. (21) reveals the influence of the wall thickness on a curved surface. For a given Biot-number, the results shown in Fig. 8 are obtained.

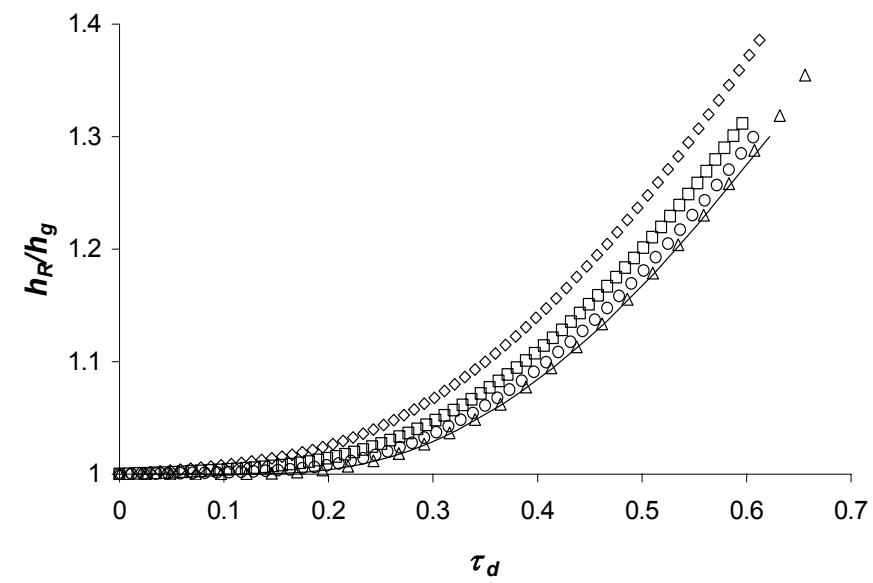

Figure 8: Effect of finite wall thickness for convex cylinder wall for $B i_{g, d}=5$ :

$-d / R=0, \quad \Delta d / R=0.13, \quad \square d / R=0.33, \quad \square \quad d / R=0.5,0 \quad d / R=0.67$

It is seen, that the behaviour is very similar to the flat plate. For thickness-to-curvature radius ratio of $d / R=0$ the values for the flat plate are obtained. The $d / R$ ratio shows little effect up to $d / R=0.33$. For higher $d / R$, Fig. 8 indicates increasing differences between Eqs. (20) and (21) for increasing $d / R$. Since the semi-infinite assumption should hold better for larger $d / R$ this is in the first view somewhat unexpected. In this investigation the radius of curvature was changed keeping the wall thickness constant. Therefore for a given time $\tau_{d}$ is constant but $\tau_{R}$ changes. The effect observed is the deviation of the approximate solution Eq. (21) from the exact solution (see Fig. 5). If one compares the time scales for curvature and thickness effects $\tau_{R}$ and $\tau_{d}$ and considers Eqs. (22) and (23) as limits for Eq. (21) to hold, one obtains:

$$
\left(\frac{d}{R}\right)_{\lim }<\sqrt{\frac{0.02}{0.25}} \approx 0.28
$$

This means that the deviation of Eq. (21) from the exact solution is the reason of the differences for larger $d / R$. Hence, 
the time criterion from the flat wall investigation obtained by Vogel and Weigand (Eq.(23)) is also valid for curved walls if the curvature effect is taken into account using Eq. (21).

\section{Backside convection}

For transient heat transfer experiments related to film cooling investigations, the influence of backside convection needs to be considered. Having a thin wall cylinder model heated from the outer wall, several conditions are possible for the backside.

Cooling the inner wall with a temperature equal to the model initial temperature would consider a typical film cooling experiment with a step change in hot gas temperature.

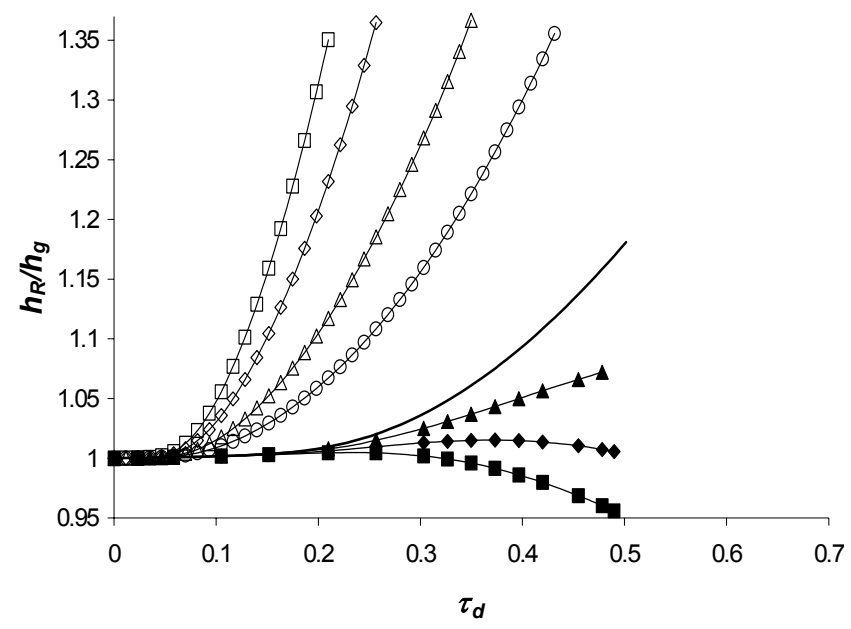

Figure 9: Effect of backside convection for $B i_{g, d}=5$ :

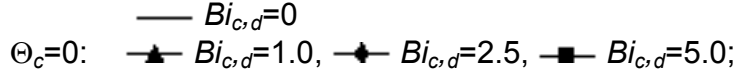

$$
\begin{aligned}
& \Theta_{c}=1: \multimap B i_{c, d}=0.5, \multimap B i_{c}, d=1.0, \multimap B i_{c}, d=2.5 \text {, } \\
& \square-B i_{c, d}=5.0 \text {; }
\end{aligned}
$$

Heating the inner wall with a temperature above the initial model temperature equal to the hot gas side temperature would relate to a heat transfer experiment with isothermal blowing, e.g. measuring the effect of film cooling blowing on heat transfer without a film cooling effect.

Having both fluid temperatures on the outer and inner walls above the model initial temperature is a typical situation for simultaneous measurements of film cooling effectiveness and heat transfer coefficient using e.g. a model insertion technique (Drost and Bölcs (1998), Falcoz (2003)).

The effect of the backside convection is addressed for different dimensionless coolant temperature and coolant side Biot-numbers using:

$$
\Theta_{c}=\frac{T_{c}-T_{i}}{T_{g}-T_{i}}, B i_{c, d}=\frac{h_{c} d}{k}
$$

For heating conditions $\left(\Theta_{c}=1\right)$ the allowable maximum test time reduces from the adiabatic case $\left(B i_{c, d}=0\right)$, with decreasing values of $\tau_{d}$ in case of increasing coolant side heat transfer coefficients. Cooling the backside $\left(\Theta_{c}=0\right)$ allows the maximum test time to be extended. The results are shown in Fig. 9.

The cases with different temperatures above the model initial temperature on both sides will lie between these two limiting cases. This is shown in Fig. 10 where for a given gas side Biot-number the value of $\Theta_{c}$ is varied. Using the comparison between the exact solution and the approximate solution helps in selecting the correct experimental parameters and to determine uncertainties introduced using the simple Eq. (21). This equation has the advantage to be straightforward implemented in existing data reduction procedures and can easily be extended to more complex situations with time varying fluid temperatures.

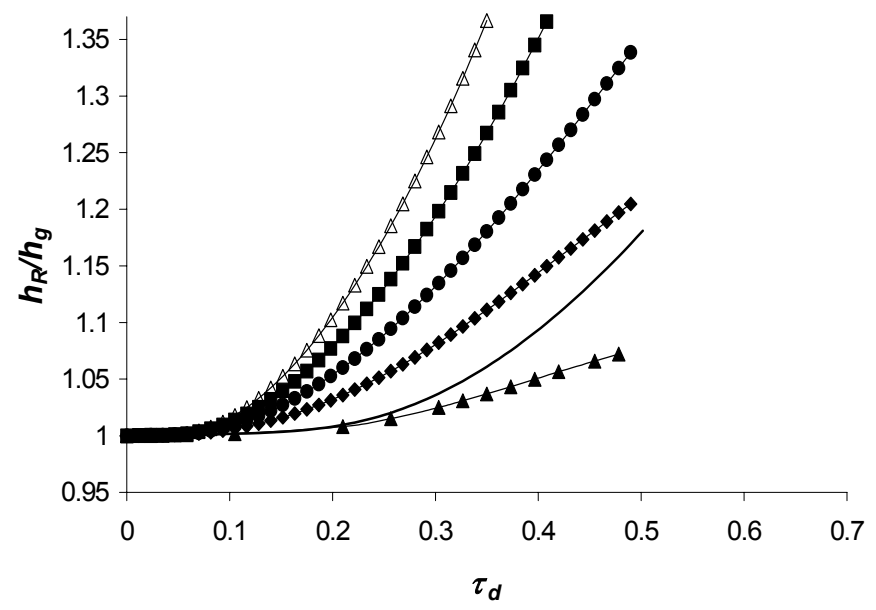

Figure 10: Effect of backside temperature condition for $B i_{g, d}=5$ : $\begin{aligned} B i_{c}, d=1.0: & B i_{c}, d=0 ; \\ -\Theta_{c}=0, \multimap \Theta_{c}=0.25, \rightarrow-\Theta_{c}=0.5, \rightarrow-\Theta_{c}=0.75, & \end{aligned}$

\section{EXPERIMENTAL RESULTS}

To verify the above theoretical considerations, heat transfer measurements were carried out on a hollow cylinder exposed to a cross flow. Tests were performed at $M a=0.11$ and $M a=0.14$ on 3 hollow cylinders with the same outer diameter but with various wall thicknesses: 2, 5 and $10 \mathrm{~mm}$. Both Mach numbers show very similar values of $N u_{D} / \sqrt{R e_{D}}$. For this reason, only the case $M a=0.14$ is presented here. The main flow and the temperature conditions are given in Table 2 while Table 3 summarizes the time duration and the dimensionless quantities of the different tests. The different heat conduction models presented above (Eqs. (1), (20) and (21)) are used to obtain the heat transfer coefficient from the wall temperature history of the transient test. 


\begin{tabular}{|c|c|c|c|c|c|c|}
\hline $\begin{array}{c}M a \\
{[-]}\end{array}$ & $\begin{array}{c}R e \\
{[-]}\end{array}$ & $\begin{array}{c}T u \\
{[\%]}\end{array}$ & $\begin{array}{c}L_{x} \\
{[\mathrm{~mm}]}\end{array}$ & $\begin{array}{c}T_{\text {tot, }} \\
{[\mathrm{K}]}\end{array}$ & $\begin{array}{c}T_{\text {tot,c }} \\
{[\mathrm{K}]}\end{array}$ & $\begin{array}{c}T_{i} \\
{[\mathrm{~K}]}\end{array}$ \\
\hline 0.14 & $86^{\prime} 600 \pm 500$ & $7 \pm 1$ & $9 \pm 1$ & 318.5 & $\sim 280$ & $\sim 268$ \\
\hline
\end{tabular}

Table 2. Main flow and temperature conditions

\begin{tabular}{|l|l|l|l|}
\hline & $\mathbf{2} \mathbf{~ m m}$ & $\mathbf{5} \mathbf{~ m m}$ & $\mathbf{1 0} \mathbf{~ m m}$ \\
\hline$t_{\max }[\mathrm{s}]$ & 23 & 35 & 40 \\
\hline$\tau_{d, \max }[-]$ & 0.602 & 0.145 & 0.042 \\
\hline$\tau_{R, \max }[-]$ & 0.011 & 0.016 & 0.019 \\
\hline$B i_{g, d}[-]$ & 3.6 & 9 & 18 \\
\hline$B i_{g, R}[-]$ & 27 & 27 & 27 \\
\hline$d / R[-]$ & 0.133 & 0.333 & 0.667 \\
\hline$\Theta_{w}[-]$ & 0.82 & 0.82 & 0.82 \\
\hline
\end{tabular}

Table 3. Time duration and dimensionless parameters for the 3 test models.

\section{Semi-infinite flat plate model}

The first approach chosen for the data reduction was to evaluate the transient tests using Eq. (1). This model, based on a flat plate geometry is simple, time effective and widely used for the transient liquid crystal technique. However, it doesn't take into account the curvature effects and the finite thickness of the wall. The $N u_{D} / \sqrt{R e_{D}}$ values obtained for the different cylinders with the semi-infinite flat plate model are compared in Fig. 11 with the correlations from Dullenkopf and Mayle (1994), van Fossen et al. (1995) and Oo and Ching (2001).

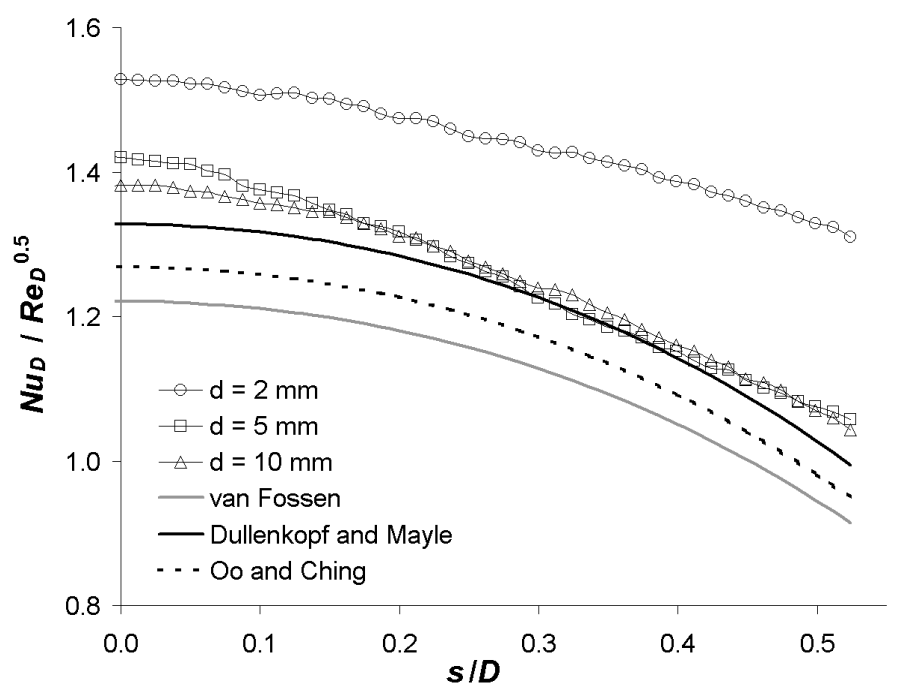

Figure 11. Heat transfer coefficient obtained using the flat plate semi-infinite model (Eq. (1)).

The cylinder with a $2 \mathrm{~mm}$ thick wall shows a heat transfer coefficient too high in comparison to the 5 and $10 \mathrm{~mm}$ test models and to the correlations. For the $2 \mathrm{~mm}$ wall $\tau_{d}$ reaches
0.602 (Table 3), $B i_{d}$ being about 3.6, Fig. 7 shows clearly that the semi-infinite assumption is not valid in this case. When the heat pulse reaches the inner surface it faces a higher thermal resistance. The rise of the temperature on the outer surface becomes then faster than for a semi-infinite solid. As a result, if the solid is assumed to be semi-infinite, the heat transfer coefficient on the outer surface is over predicted for long measurement time. For the 5 and $10 \mathrm{~mm}$ thick walls, $\tau_{d}$ reaches only 0.145 , respectively 0.042 and Eq. (23) is respected. In this case, the finite thickness of the wall has no influence on the temperature evolution on the outer surface. The cylinder with 5 and $10 \mathrm{~mm}$ walls give then very similar heat transfer coefficients.

However, $N u_{D} / \sqrt{R e_{D}}$ of the 5 and $10 \mathrm{~mm}$ thick hollow cylinders are still too high compared to the correlations because the curvature is not taken into account in Eq. (1). For a convex body, the surface available for heat conduction in the direction normal to the test surface becomes smaller as the heat penetrates deeper into the solid. As a matter of fact, the thermal resistance increases and the evolution of the surface temperature during the transient test is faster than it would be on a flat body. Assuming a flat surface for a convex test model results in over-predicting the heat transfer coefficient . In the present case, according to Table 3 and Fig. $4, h_{f p}$ is about $7 \%$ higher than the correct value.

The errors of the experimental data showed in Fig. 11 are due to both, the errors in the measurement of the experimental parameters and non-consideration of finite thickness and curvature effects in the data analysis.

\section{Semi-infinite model with approximative curvature correction}

The measured data was also analyzed using the simplified model from Buttsworth and Jones (1997), that is to say with Eq. (21) instead of Eq. (1). The results obtained for the 3 test cylinders are compared in Fig. 12 with the correlations.

In comparison to the results of Fig. 11, Eq. (21) shifts the heat transfer coefficient towards smaller values and brings it closer to the correlations. The heat transfer coefficient obtained for the 5 and $10 \mathrm{~mm}$ thick cylinders is in good agreement with the correlations. As shown in Fig. 5, Eq. (21) accurately takes into account the curvature effects for $\tau_{R}<0.02$ which is the case here. $N u_{D} / \sqrt{R e_{D}}$ resulting from the measured data of the 2 $\mathrm{mm}$ thick cylinder is still too high since the semi-infinite assumption is not valid for this test model. Fig. 7 shows that in this case the semi-infinite assumption provides a heat transfer coefficient about $25 \%$ too high. The errors of the experimental results from Fig. 12 are due to the errors in the measurement of the experimental parameters and for the $2 \mathrm{~mm}$ thick cylinder to the non-consideration of finite thickness effects in the data analysis. 


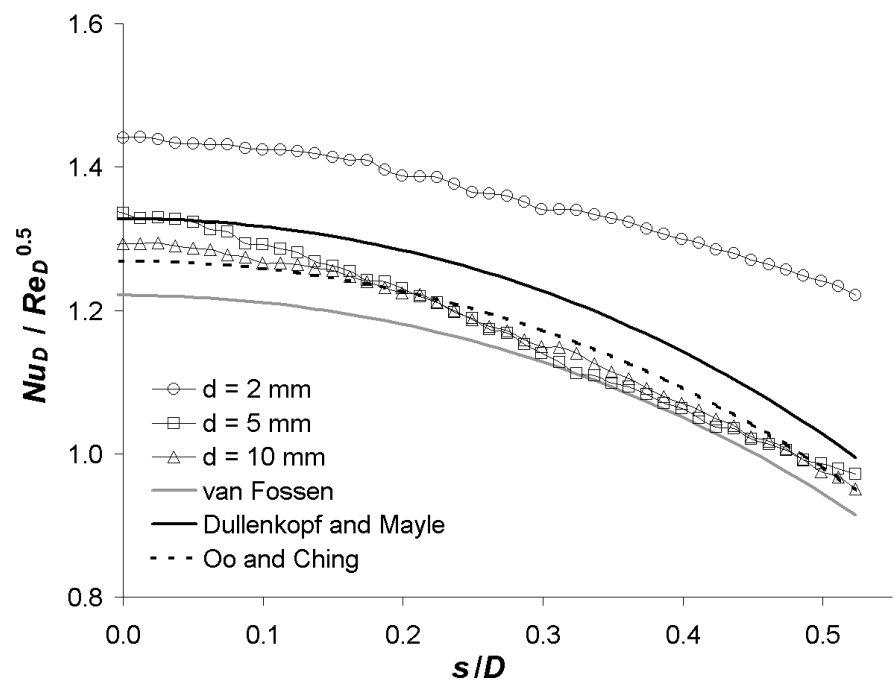

Figure 12. Heat transfer coefficient obtained using the approximate model (Eq. (21)).

\section{Hollow cylinder model}

Finally, the exact analytical solution (Eq. (20)) was used for the data reduction of the tests. Both curvature effects and finite wall thickness are here taken into account, but the calculations are much more time consuming than for the two models discussed above. The $N u_{D} / \sqrt{R e_{D}}$ results are presented in Fig. 13 together with the correlations.

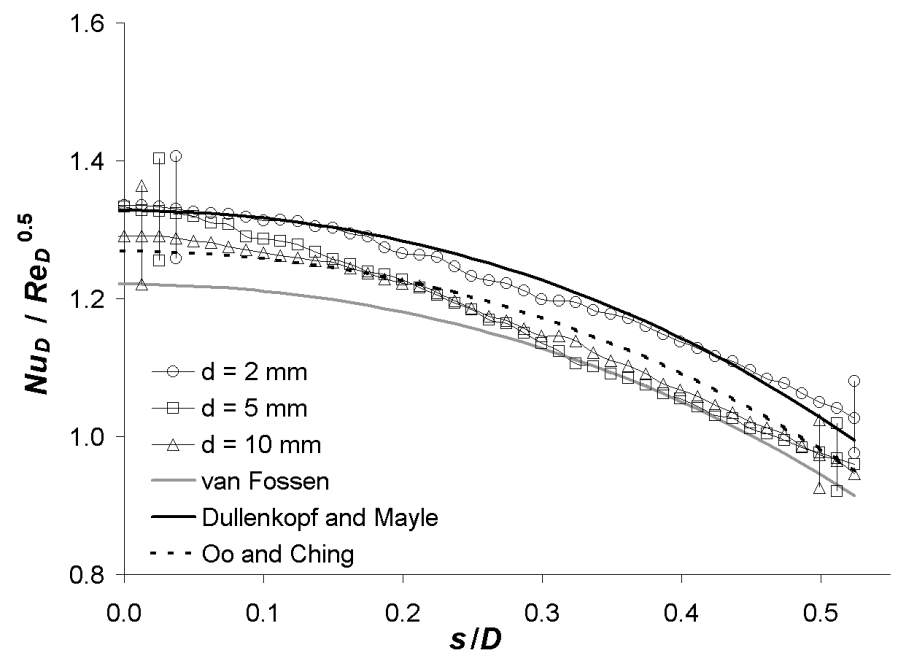

Figure 13. Heat transfer coefficient obtained using the exact solution (Eq. (20)).

The heat transfer coefficients obtained for the cylinders with 5 and $10 \mathrm{~mm}$ thick walls hardly change between Fig. 12 and Fig. 13. This confirms that, for those two cylinder models, both the semi-infinite assumption and the simple curvature correction from Buttsworth and Jones (Eq. (21)) are correct. With the exact analytical heat conduction model, the measurements on the cylinder with a $2 \mathrm{~mm}$ thick wall give now reliable results that are in good agreement with the correlations. The differences between the 3 cylinder models are less than $10 \%$. As both curvature and finite thickness effects are here taken into account the data analysis is correct. The uncertainties of the experimental data are thus only due to the errors in the measurement of the experimental parameters. The measurement uncertainties are given by the error bars added at $\mathrm{s} / \mathrm{D}=0$ and 0.5 .

For the cylinders with 5 and $10 \mathrm{~mm}$ thick walls, the semiinfinite model with the simple correction from Buttsworth and Jones (Eq. (21)) is sufficient to give accurate results, while the exact analytical solution (Eq. (20)) is needed for the $2 \mathrm{~mm}$ thick wall model in order to take into account the finite wall thickness.

\section{CONCLUSION}

A new data analysis method for transient liquid crystal measurements is presented. The new analysis is based on the equation of heat conduction in a hollow cylinder and exactly accounts for curvature and finite wall thickness effects.

Comparisons with the classical semi-infinite flat plate approach and with the simplified curvature correction from Buttsworth and Jones (1997) showed that curvature effects and the finite thickness of the wall can have significant influence in transient heat transfer experiments.

To verify the analytical results, transient liquid crystal measurements were evaluated with the different analysis methods. The experimental results validated the analytical considerations and showed good agreement with recent heat transfer correlations.

\section{REFERENCES}

Baughn, J. W., Ireland P. T., Jones, T. V. and Saniei, N., 1989, "A Comparison of the Transient and Heated-Coating Methods for Measurement of Local Heat Transfer Coefficients on Pin Fin,” ASME Journal of Heat Transfer, 111, pp. 877-881.

Butler, R. J., and Baughn, J. W., 1994, "Validation of an In-Situ Heated Transient Technique with Local Heat Transfer Measurements on a Cylinder in Crossflow," 6th AIAA/ASME Thermophysics and Heat Transfer Conference, AIAA paper 942009.

Buttsworth, D. R., and Jones, T. V., 1997, "Radial Conduction Effects in Transient Heat Transfer Experiments," The Aeronautical Journal, pp. 209-212.

Carslaw, H. S., and Jaeger, J. C., 1992, "Conduction of Heat in Solids," $2^{\text {nd }}$ ed., Oxford Science Publications, Clarendon Press, Oxford, UK.

Clifford, R. J., Jones, T. V., and Dunne, S. T., 1983, "Techniques for Obtaining Detailed Heat Transfer Coefficient Measurements within Gas Turbine Blade and Vane Cooling Passages," ASME paper 83-GT-58. 
Drost, U., and Bölcs, A., 1998, "Investigation of Detailed Film Cooling Effectiveness and Heat Transfer Distributions on a Gas Turbine Airfoil," ASME Paper 98-GT-20.

Dullenkopf, K., and Mayle, R. E., 1994, “An Account of Free-Stream-Turbulence Length Scale on Laminar Heat Transfer," ASME paper 94-GT-174.

Ekkad, S. V., Han, J. C. and Du, H., 1998, "Detailed Film Cooling Measurements on a Cylindrical Leading Edge Model: Effect of Free-Stream Turbulence and Coolant Density," ASME Journal of Turbomachinery, 120, pp. 799-807.

Ekkad, S. V., and Han, J. C., 2000, "A Transient Liquid Crystal Thermography Technique for Gas Turbine Heat Transfer Measurements," Measurement Science and Technology, 11, pp. 957-968.

Falcoz, C., 2003, "A Comparative Study of Showerhead Cooling Performance," Ph.D. Thesis n²735, EPFL, Switzerland.

Guo, S. M., Spencer, M. C., Lock, G. D., Jones, T. V., and Harvey, N.W., 1995, "The application of Thin Film Gauges on Flexible Plastic Substrates to the Gas Turbine Situation," ASME paper, 95-GT-357.

Ireland, P. T., and Jones, T. V., 1985, "The Measurements of Local Heat Transfer Coefficients in Blade Cooling Geometries," AGARD Conference Proceedings on Heat Transfer and Cooling, CP 390 Paper 28, Bergen.

Ireland, P. T., 1987, "Heat Transfer in Gasturbines," Ph.D. Thesis, Oxford University.

Ireland, P. T., and Jones, T. V., 2000, "Liquid Crystal Measurements of Heat Transfer and Surface Shear Stress," Measurements Science and Technology, 11, pp. 969-985.

Oo, A. N., and Ching, C. Y., 2001, "Effect of Turbulence With Different Vortical Structures on Stagnation Region Heat Transfer," ASME Journal of Heat Transfer, 123, pp. 665-674.

Reiss, H., 2000, "Experimental Study on Film Cooling of Gas Turbine Airfoils Using Shaped Holes," Ph.D. Thesis $\mathrm{n}^{\circ} 2209$, EPFL, Switzerland.

Schultz, D. L., and Jones, T. V., 1973, "Heat transfer measurements in short duration hypersonic facilities," NATO Advisory Group Aeronautical RD AGARDOGRAPH, 165.

Van Fossen, G. J., Simoneau, R. J., and Ching, C. Y., 1995, "Influence of Turbulence Parameters, Reynolds Number, and Body Shape on Stagnation Region Heat Transfer," ASME Journal of Heat Transfer, 117, pp. 598-603.

Vogel, G., Weigand, B., 2001, “A New Evaluation Method for Transient Liquid Crystal Experiments," National Heat Transfer Conf., NHTC2001-20250, California, USA.

Wang, Z., Ireland, P. T., Kohler, S. T., and Chew, J.W., 1996, "Heat Transfer Measurements to a Gas Turbine Cooling Passage with Inclined Ribs," ASME paper, 96-GT-542. 